\title{
Medium and Long Term Voltage Stability Based on Decoupled Algorithm
}

\author{
Xiaoying Zhang ${ }^{1, \text { a }}$, Kun Wang ${ }^{1, b}$,Zheng Jiang ${ }^{1, c}$ \\ ${ }^{1}$ School of Electrical and Information Engineering, Lanzhou University of Technology, Lanzhou, \\ 730050, China
}

aemail: 245659219@qq.com, bemail:471943808@qq.com, cemail:jzheng@163.com

Keywords: voltage stability; time-domain analysis; decoupled algorithm; quasi-steady-state(QSS). Abstract: Considering the characteristic of medium and long term voltage stability, the simulation algorithm of medium and long term voltage stability used quasi-steady-state assumption is studied. Based on the numerical integration methods currently used in the time-domain simulation analysis, a decoupled simulation algorithm which synthesizes the desirable properties of implicit and explicit algorithms is presented. For quasi-steady-state simulation of the system, its algebraic equations and differential equations are solved separately. This algorithm effectively coordinates the contradictions in both computational speed and accuracy of the time-domain simulation of the medium and long term voltage stability. Then the implement method of the quasi-steady-state simulation based on the continuous load parameters is discussed, and the simulation results are illustrated on the New England 10-machine 39-bus test system, by which the accuracy and effectiveness of the method are approved.

\section{Introduction}

Voltage stability of power system refers to the ability that with given initial conditions and disturbances, the power system remains stable on all bus voltages, which depends on the ability of preserving or restoring load demand and load balance. Voltage stability of power system was put forward by Malkovich in the 1940s [1], and as the continuous expansion of grid, the system voltage stability problem becomes increasingly serious. In the modern power system voltage stability analysis, people put forward various methods based on continuous algorithm to study the steady state of voltage stability issues. However, we still need to capture the transient response of time-domain simulation and grasp the timing of the control system operation. Time domain response can capture the change of system unstable process, and then put forward the control sequence. But in order to capture the transient response of the system, we need to solve a set of differential algebraic equations (DAE). Typical power system network contains a lot of dynamic and static components, and each of these individual components may need to be represented by a number of differential algebraic equations. Therefore, for a real power system, the number of required differential algebraic equations is very large.

For complex power system dynamic and static components, when its time constant changed in a larger scope, it would lead to stiffness problem [2]. Currently, the numerical method used in time domain simulation produces wrong results for rigid system, mainly because the accumulation of errors from numerical integration step by step process [3]. Numerical integral method is usually used in the time domain simulation analysis, and the integral methods can be separated into two categories: explicit algorithm and implicit algorithm. The explicit algorithm involves fixed point iteration with a faster arithmetic speed, but there are numerical stability problems in dealing with rigid problem. The implicit algorithm is more stable, but arithmetic speed is slower. Some papers pointed out that obviously reducing control step is needed to keep the numerical stability in the case of using explicit algorithm, or explained the difference between explicit and implicit algorithm for a rigid system through a simple example of two variables. At the same time, some algorithms were proposed to solve the rigid problem [4,5]. In this paper, on the basis of numerical integral method which currently used in the time domain simulation analysis, a decoupled quasi-steady-state (QSS) simulation algorithm which synthesizes the desirable properties of implicit and explicit algorithms is put forward. Taking advantage of the decoupled time domain simulation method, the realization of 
quasi-steady-state simulation is discussed under the condition of introducing technology of load parameters continuous change. Finally, New England 39-bus system is used to verify the validity of this simulation method.

\section{Decoupled QSS simulation method}

In order to capture the response of the medium and long term voltage stability, people proposed many efficient algorithms to reduce the amount of calculation, and the QSS time-domain simulation method is the most widely used [6]. The QSS simulation is approximately based on the assumption that there are stable equilibrium of short-term dynamic process, short-term dynamic process gets to the balance point in an enough short time. QSS simulation is a powerful tool for medium and long term voltage stability analysis.

For the rigidity problem of the power system, we can use the implicit algorithms to keep the step length control of the numerical stability. But, because the implicit algorithms are usually used to simulate the dynamic characteristics, each integral step of a stiff equation involves a solving process of nonlinear equations, which leads to a set of linear problems[7]. As a result, the algorithms for solving the rigid systems take a lot of time to solve the system linear equations. Therefore, for the QSS simulation of stiff systems, we put forward a decoupled method which solves the system algebraic equations and differential equations or difference equations are separately. If a problem can be divided into a rigid part and a nonrigid part as shown in Eq. 1:

$$
\left\{\begin{array}{l}
\dot{x}_{s}=f_{s}\left(x_{s}, x_{n}\right) \\
\dot{x}_{n}=f_{n}\left(x_{s}, x_{n}\right)
\end{array}\right.
$$

where $x_{s}, x_{n}$ represent rigid and nonrigid variables, and $f_{s}, f_{n}$ represent rigid and nonrigid equations. Then, we can treat this system as two parts: implicit algorithms for the rigid parts and explicit algorithms for the nonrigid parts.

In the view of geometry, the solutions of the ODE and DAE systems are points or vectors in the multi-dimensional space. This space can be divided into two or more subspaces, and the solution vectors can be decomposed into corresponding sub-vectors in each subspace. Thus, by dividing the space into several subspaces, the solution vectors can be decomposed into sub-vectors, so the original ODE and DAE systems can be decoupled into several small dimension systems.

We suppose $\mathrm{P}$ be the invariant subspace corresponding to the $\mathrm{m}$ eigenvalues which are outside the stability domain of an explicit method and let $Z_{1}$ be an orthonormal basis in P. Therefore, $Z_{1}$ is an $\mathrm{n} \times \mathrm{m}$ matrix which meets the following condition:

$$
Z_{1}^{T} Z_{1}=I_{m}
$$

where $I_{m}$ is an $\mathrm{m} \times \mathrm{m}$ unit matrix.

Besides, there is a orthogonal complement $\mathrm{Q}$, if let $Z_{2}$ be the standard quadrature basis in $\mathrm{Q}$, then

$$
Z_{2}^{T} Z_{2}=I_{n-m}
$$

Because $\mathrm{Q}$ is a orthogonal complement of $\mathrm{P}$, there are:

$$
Z_{1}^{T} Z_{2}=0 \quad Z_{2}^{T} Z_{1}=0
$$

Hence, the space can be represented the sum of $\mathrm{P}$ and $\mathrm{Q}$, where $Z_{1}$ and $Z_{2}$ are the internal standard

orthonormal basis respectively, and the matrixes $Z_{1} Z_{1}^{T}$ in $\mathrm{m} \times \mathrm{m}$ dimension and $Z_{2} Z_{2}^{T}$ in (n-m) $\times(\mathrm{n}-\mathrm{m})$ dimension can be regarded as the orthogonal projectors in the two subspaces, so any vector in the full space can be projected into the two subspaces by coordinating the multiple projection.

As the original n-dimensional space vectors can be decomposed into the sum of two small dimension vectors, the original system can be divided into two subsystems: 


$$
\left\{\begin{array}{l}
f^{P}(p, q)=Z_{1}^{T} f\left(Z_{1} p+Z_{2} q\right) \\
f^{Q}(p, q)=Z_{2}^{T} f\left(Z_{1} p+Z_{2} q\right)
\end{array}\right.
$$

and the ODE system equations can be decoupled into two systems:

$\left\{\begin{array}{l}\dot{p}=f^{P}(p, q)=Z_{1}^{T} f\left(Z_{1} p+Z_{2} q\right) \\ \dot{q}=f^{Q}(p, q)=Z_{2}^{T} f\left(Z_{1} p+Z_{2} q\right)\end{array}\right.$

Having solved the above decoupled equations, vectors p and q can be calculated separately, so the initial state of the system can be represented as $x=Z_{1} p+Z_{2} q$.

Eq. 6 has the expected form similar to Eq. 1, and all the eigenvalues of the second equation are in the stability domain. Therefore, an explicit algorithm can be used to solve the second set of equations, while an implicit algorithm can be used to solve the first set of equations, thereby coordinates the contradictions in both computational speed and accuracy of the time- domain simulation of the medium and long term voltage stability effectively.

\section{System simulation examples}

The single line diagram of New England 10-machine 39-bus test system is used for simulation. The full explicit forward Euler algorithm and full implicit trapezoidal algorithm mentioned above can be applied to this system. The generators in the test system adopt biaxial mode provided in [6]. and the exciters and governors are consistent with those mentioned in [8]. In this case, there are 9 states for each generator, and the total number of differential states and algebraic states are 90 and 78 respectively. The step size during the simulation is chosen as $0.025 \mathrm{~s}$, and the stiff invariant subspace is calculated under the initial state with 19 dimensions. For the decoupled method, the dimension of the nonlinear equation system is 97 , while the dimensions of the nonlinear systems are 78 and 168 for the full explicit method and full implicit method respectively.

The selected contingency is the transmission line trip between bus 6 and bus 7 at $0.05 \mathrm{~s}$, and the simulation duration is 20s. The simulations adopt full explicit algorithm, full implicit algorithm and decoupled algorithm respectively, and the results are shown in Fig. 1, Fig. 2 and Fig. 3.

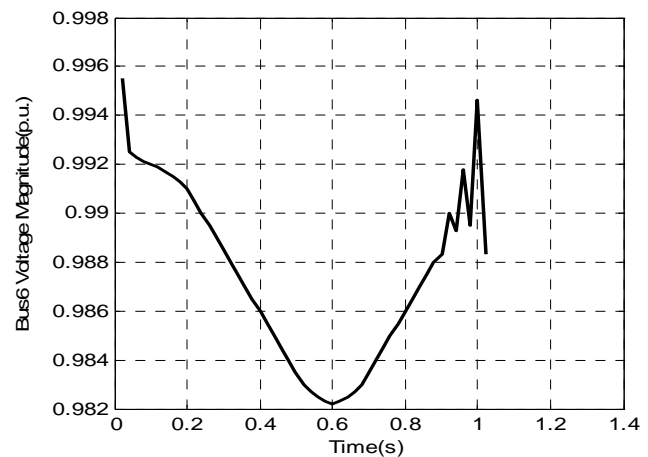

Fig.1 Simulation result by full explicit algorithm

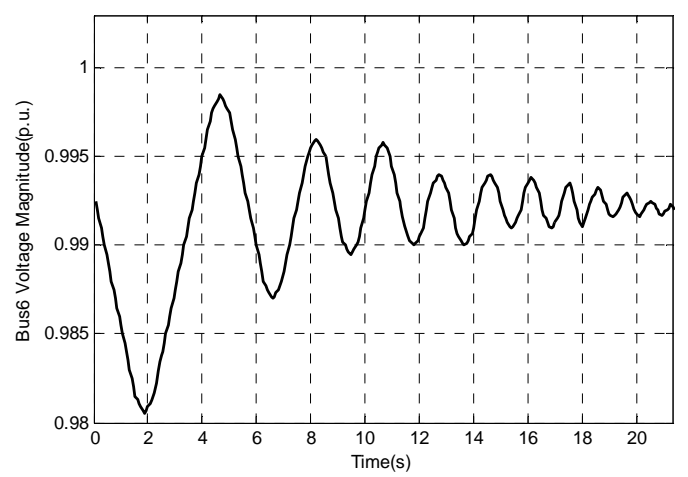

Fig.3 Simulation result by decoupled algorithm

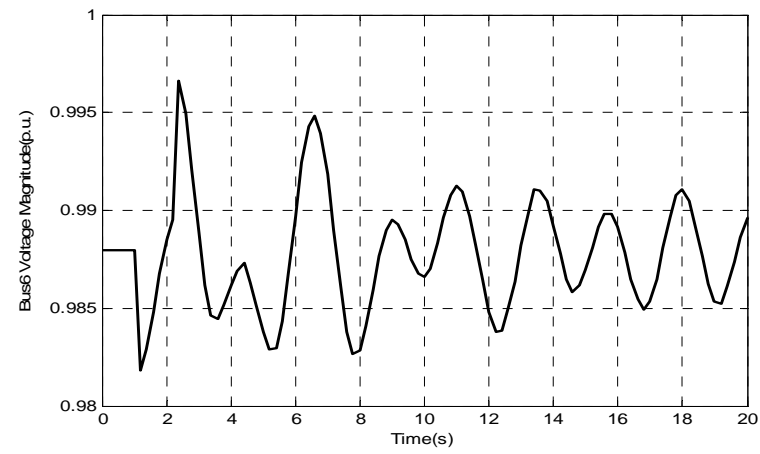

Fig.2 Simulation result by full implicit algorithm

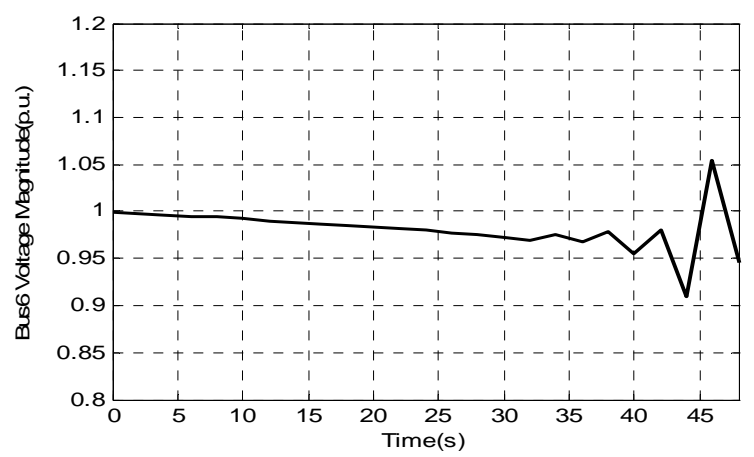

Fig.4 Mid and long-term simulation result 
In the actual, the response after disturbance should make the system maintain its stability, but the simulation results show that the decoupled algorithm and the full implicit algorithm produce stable results, however, the full explicit algorithm fails to give the correct outcome.

From Fig. 1, the result of full explicit algorithm (using pure forward Euler algorithm) appears bifurcation at 1s, which leads to the incapable of dynamic simulation. Before the explicit algorithm bifurcation, an oscillatory behavior can be observed which is caused by the numeric value and does not represent the actual system response.

Meanwhile, the simulation results of full implicit algorithm and decoupled algorithm provide the stable system response respectively shown in Fig. 2 and Fig. 3. Both algorithms give the steady state response after the system disturbance, and the results of the two algorithms match the system running results very well. From the simulation results, the system transient process will subside soon after the disturbance, and reaches a new transient equilibrium. But comparing with the algorithms of the two simulation diagrams, the decoupled algorithm simulation curve shown in Fig. 3 is more ideal than the full implicit algorithm simulation curve shown in Fig. 2.

Set the load of bus 31 which connected with bus 6 through transformer increases at a rate of $0.5 \%$ per second to capture the response of the long-time unstable system, and the simulation result of bus 6 voltage is shown in Fig. 4. Through the result, decoupled algorithm combines the advantages of both full explicit algorithm and full implicit algorithm, in another word, it gets the numerical stability as well as the good efficiency. When using this decoupled algorithm, the rigid and nonrigid subspace are separated and processed with different algorithms.

\section{Conclusion}

This paper studies simulation algorithm of medium and long term voltage stability based on the quasi-steady-state assumption, and based on the numerical integration methods currently used in the time domain simulation analysis, a new decoupled simulation algorithm whose algebraic equations and differential equations or difference equations are solved separately is put forward for quasi-steady-state simulation of the system. This algorithm combines the expectation features of explicit and implicit algorithm, not only the stability of the numerical calculation result is ensured, but also a good operational efficiency is obtained.

\section{Acknowledgements}

This work was financially supported by the Natural Science Foundation of China (No. 50967001) and Excellent Youth Teacher Foundation of Lanzhou University of Technology (No. Q200814).

\section{References}

[1] Yongchun Su, and Shijie Cheng: Elect. Power Automat. Eqpt. Vol. 26 (2006), p. 97(in Chinese).

[2] Z. Feng, V. Ajjarapu, and D.J. Maratukulam: IEEE Trans. Power Syst. Vol. 15 (2000), p.342.

[3] Taishan Xu, Yanhong Bao, Yusheng Xue, and Yi Chen: Automat. Elect. Power Syst. Vol. 24 (2000), p. 9(in Chinese).

[4] Fang Cao and Wei Wang: Int. J. of Modelling, Identification and Control, Vol.15 (2012), p. 20.

[5] Yifei Wu, Fangqin Huang, Guoping Wei, and Yu Guo: Int. J. of Modelling, Identification and Control, Vol.19 (2013), p. 361.

[6] Yangyu Hu, Tianguang Lv, Shuangwei Chu, Ning An, Hongjun Fu, and Jianhua Sun: Power Syst. Tech. Vol. 36 (2012), p. 157(in Chinese).

[7] Qin Wang and V. Ajjarapu: IEEE Trans. Power Syst. Vol. 20 (2005), p. 516. 
[8] Ning An, Xinglei Chen, Rui Ren, and Shuangxi Zhou: Power System Protection and Control Vol. 39 (2011), p. 1(in Chinese). 